\title{
Neovascular Glaucoma Associated with Chronic Rhegmatogenous Retinal Detachment
}

\section{Dong lk Kim}

HanGil Eye Hospital

Se Joon Woo ( $\sim$ sejoon1@snu.ac.kr)

Seoul National University Bundang Hospital, Seoul National University College of Medicine

\section{Research Article}

Keywords: angiography, chronic retinal detachment, neovascular glaucoma, nonperfusion, rhegmatogenous retinal detachment

Posted Date: January 7th, 2022

DOI: https://doi.org/10.21203/rs.3.rs-1190159/v1

License: (c) (i) This work is licensed under a Creative Commons Attribution 4.0 International License. Read Full License 


\section{Abstract \\ Background}

To demonstrate the clinical features and natural course of chronic retinal detachment associated neovascular glaucoma.

\section{Methods}

Ten patients, diagnosed with chronic retinal detachment-associated neovascular glaucoma during 2007-2016 were retrospectively investigated. Besides chronic retinal detachment, no patients had any neovascular glaucoma-predisposing conditions, such as carotid artery disease. Retinal perfusion status was evaluated from postoperative visual acuity, intraocular pressures, ocular examination findings, and fluorescein angiography images.

\section{Results}

The mean age of patients was 57.5 (range: 22-78) years. Complete retinal reattachment was achieved in 3 eyes, while partial or total chronic retinal detachment persisted in 7 eyes. Wide-angle fundus fluorescein angiography revealed peripheral retinal capillary obstruction and severe non-perfusion. Neovascular glaucoma developed 213.4 months (17-634 months) after retinal detachment. Three eyes received Ahmed valve implantation, while 5 eyes received intravitreal bevacizumab injection. Intraocular pressure was controlled in 10 eyes. Two eyes developed phthisis bulbi during follow-up.

\section{Conclusions}

In eyes with a chronic retinal detachment history, iris neovascularization and neovascular glaucoma can develop due to retinal capillary obstruction and chronic retinal ischemia, even after achieving retinal reattachment. We suggest routine follow-up examinations for patients with chronic retinal detachment, particularly for eyes with retinal non-perfusion, as detected on fundus fluorescein angiography.

\section{Background}

Neovascularization of the retina and iris and resultant neovascular glaucoma is a common consequence of chronic retinal ischemic diseases. The new vessels grow at the pupillary border and iris surface (neovascularization of the iris [NVI]) and over the iris angle (neovascularization of the angle [NVA]). These NVI and NVA form fibrovascular membranes, which progressively obstruct the trabecular meshwork. As the disease progresses, the fibrovascular membranes mature and contract, and pull the iris toward the trabecular meshwork. These processes generate peripheral anterior synechiae and progressive synechial angle closure, and cause secondary angle-closure glaucoma or neovascular glaucoma (NVG) [1].

Proliferative diabetic retinopathy, central retinal vein occlusion, central retinal artery occlusion, and ocular ischemic syndrome can cause neovascularization of intraocular structures as well as NVG. However, the development of ocular neovascularization as a complication of rhegmatogenous retinal detachment (RRD) has rarely been reported [2,3]. There have been a few reports of fluorescein angiography of RRD showing capillary non-perfusion in the detached retina area $[2,4-6]$. Vitreous vascular endothelial growth factor levels in eyes with retinal detachment have been reported to be significantly higher than in samples from control patients [7].

In this retrospective case series, we present 10 eyes from 10 patients with NVG, who had no NVG-predisposing factors other than long-standing RRD.

\section{Methods}

This was a retrospective study that included patients who visited Seoul National University Bundang Hospital between 2007 and 2016 . This study was approved by the institutional review board of Seoul National University Bundang Hospital (IRB no. B-2112-725-105) and followed the tenets of the Declaration of Helsinki. Since all data collected were retrospective, the requirement of the informed consent was waived by the IRB of Seoul National University Bundang Hospital. Patients were included if they had a history of retinal detachment and NVI or NVG. Patients who had predisposing conditions for intraocular neovascularization, such as carotid artery disease, proliferative diabetic retinopathy, retinal vein occlusion, or ocular ischemic syndrome, were excluded.

A total of 10 eyes of 10 patients were included in the study. Data on medical history and clinical features, including visual acuity (VA), intraocular pressure (IOP), slit lamp examination, fundus examination, fundus fluorescein angiography, and treatment methods were obtained.

\section{Results}

There were 5 males, and 5 females. The mean age of all patients was 57.5 years (range: 22-78 years). All patients had unilateral NVG, with the involvement of 3 right and 7 left eyes. No patients had diabetes, and 4 patients had hypertension, which was well-controlled by medication. No patients had evidence of any systemic disease accounting for the retinal neovascularization, such as carotid artery stenosis, or a family history of vascular disease (Table 1). 
Table 1

Demographic and systemic characteristics of all patients included in this case series

\begin{tabular}{|llllll|}
\hline Case & Age & Sex & DM & HTN & Other risk factors \\
\hline 1 & 60 & Male & - & - & Smoking \\
\hline 2 & 36 & Male & - & - & \\
3 & 78 & Male & - & - & \\
\hline 4 & 65 & Female & - & + & Dyslipidemia \\
\hline 5 & 52 & Male & - & + & \\
\hline 6 & 46 & Female & - & - & \\
7 & 22 & Female & - & - & \\
\hline 8 & 78 & Male & - & + & \\
\hline 9 & 78 & Female & - & + & \\
\hline 10 & 60 & Female & - & - & Dyslipidemia \\
\hline Abbreviations: DM Diabetes mellitus, HTNHypertension
\end{tabular}

The ophthalmologic features of the patients are presented in Table 2. Initial VA was poor in all patients. There was no light perception in 3 patients (Cases 1, 2, and 6), light perception only in 2 patients (Cases 5 and 9), hand motion in 3 patients (Cases 3, 7, and 8), VA of 20/400 in 1 patient (Case 4), and of 20/1000 in 1 patient (Case 10), based on Snellen charts. Three patients had poor vision from birth, and they were diagnosed as having spontaneous resolution of chronic retinal detachment (Cases 1, 2, and 5). Retinal detachment was diagnosed at year 1991 in 1 patient (Case 6 ), 2002 in 1 patient (Case 7), 2007 in 3 patients (Case 3, 8, and 9), and 2011 in 2 patients (Cases 4 and 10). Three patients did not have a history of vitreoretinal surgery; 6 patients had pars plana vitrectomy, and 3 patients underwent scleral buckling for retinal detachment.

Table 2

Ophthalmologic features of eyes included in this case series

\begin{tabular}{|c|c|c|c|c|c|c|c|c|c|c|c|}
\hline Case & $\begin{array}{l}\text { Initial } \\
\text { VA }\end{array}$ & $\begin{array}{l}\text { Final } \\
\text { VA }\end{array}$ & $\begin{array}{l}\text { Initial } \\
\text { IOP } \\
(\mathrm{mmHg})\end{array}$ & $\begin{array}{l}\text { Peak } \\
\text { IOP } \\
(\mathrm{mmHg})\end{array}$ & $\begin{array}{l}\text { Final IOP } \\
(\mathrm{mmHg})\end{array}$ & $\begin{array}{l}\text { Management } \\
\text { of RRD }\end{array}$ & $\begin{array}{l}\text { Management } \\
\text { of NVG }\end{array}$ & $\begin{array}{l}\text { Time } \\
\text { from } \\
\text { RRD to } \\
\text { NVI } \\
\text { (months) }\end{array}$ & $\begin{array}{l}\text { Follow } \\
\text { up } \\
\text { (months) }\end{array}$ & Phthisis & $\begin{array}{l}\text { Retinal } \\
\text { reattachment }\end{array}$ \\
\hline 1 & NLP & NLP & 62 & 62 & 14 & Observation & $\begin{array}{l}\text { IVB, AVI, } \\
\text { scatter laser, } \\
\text { eyedrop }\end{array}$ & 634 & 78 & - & + \\
\hline 2 & NLP & NLP & 48 & 48 & 8 & Observation & AVI, eyedrop & 340 & 69 & - & - \\
\hline 3 & $\mathrm{HM}$ & NLP & 50 & 42 & 22 & SB, PPV & $\begin{array}{l}\text { Iridoplasty, } \\
\text { eyedrop }\end{array}$ & 18 & 56 & - & - \\
\hline 4 & $20 / 400$ & $\mathrm{HM}$ & 8 & 36 & 18 & PPV & IVB, eyedrop & 17 & 51 & - & - \\
\hline 5 & LP & NLP & 18 & 42 & 3 & Observation & Eyedrop & 608 & 22 & + & - \\
\hline 6 & NLP & NLP & 45 & 45 & 24 & SB & Eyedrop & 216 & 15 & - & - \\
\hline 7 & $\mathrm{HM}$ & NLP & 43 & 43 & hypotony & PPV & IVB, eyedrop & 77 & 87 & + & + \\
\hline 8 & $\mathrm{HM}$ & LP & 39 & 39 & 20 & PPV, SB & AVI, eyedrop & 88 & 9 & - & + \\
\hline 9 & LP & LP & 31 & 31 & 3 & PPV & IVB, eyedrop & 88 & 1 & - & - \\
\hline 10 & $20 / 1000$ & LP & 8 & 29 & 7 & PPV & IVB, eyedrop & 48 & 51 & - & - \\
\hline
\end{tabular}

Five patients had NVI and had high IOP at the initial presentation (Fig. 1). During follow up, NVI as well as IOP elevation were observed in all patients. All patients were diagnosed as having NVG. The mean time from onset of RRD to the onset of NVI or NVG was 213.4 months. (17-634 months). In 3 patients, NVG occurred even after achieving reattachment of the detached retina (Figs. 2, 3). Wide-angle fluorescein angiography was performed in 2 cases (Cases 1 and 8), both of which showed wide areas of retinal capillary obstruction as well as retinal vascular leakage in the reattached retina. Interestingly, the retinal capillary obstruction was also present in the non-detached retina. 
To control high IOP, Ahmed valve implantation was performed in 3 eyes, and intravitreal bevacizumab injection was performed in 5 eyes. All patients used topical IOP lowering medications. Prophylactic scatter laser photocoagulation was performed in 1 eye (Case 1), because of the wide retinal nonperfusion seen on fluorescein angiography. IOPs were controlled in 10 of 10 eyes. However, two eyes developed phthisis bulbi during follow-up (Cases 5 and 7).

\section{Discussion}

Our study showed that NVG can develop in eyes with chronic retinal detachment, even in the absence of predisposing conditions, such as carotid artery diseases. Rarely, retinal detachment can cause NVI and NVG [8-10]. A previous study [9] has reported that NVI is associated with chronic retinal detachment. They revealed that risk factors for NVI are an age $\geq 50$ years, severe myopia, a history of increased IOP, a history of choroidal detachment, and a large scleral buckle. In that study, 6 of 30 patients showed improvement of NVI after reattachment. Another study [8] revealed that new retinal vessels were totally regressed within 15 days to 3 months after retinal reattachment, in 9 of 9 eyes. However, our cases showed persistent NVI and experienced NVG even after successful retinal reattachment.

The pathogenic mechanism underlying development of NVI and NVG most likely involves retinal capillary obstruction and retinal ischemia, as shown by wide-angle fluorescein angiography in our cases. However, it is unclear why retinal capillary obstruction occurs even in the attached retina. Postoperative inflammation and retinal vasculitis may be the cause of retinal vascular obstruction, but this should be further investigated. In previous studies, it was considered that the new peripheral retinal vessels formed secondary to retinal hypoxia due to reduced retinal blood flow in the detached retina. Persistent retinal detachment, reduction of choroidal circulation, and retinal circulation was thought to play roles in ocular neovascularization [8, 9]. In addition, as posterior vitreous detachment usually occurred in eyes with retinal detachment, neovascularization would develop in the anterior segment, rather than in the posterior segment, resulting in NVG.

It is noteworthy that NVG occurred even after achieving reattachment of a detached retina in our cases. Fluorescein angiography showed diffuse widespread non-perfusion areas and leakage in the reattached retina. Therefore, regular follow-up examinations, including careful inspection of the NVI, as well as NVA are recommended for patients who have a history of chronic retinal detachment. In cases that failed to achieve retinal reattachment, even more caution should be taken, and patients should be informed of the possibility of developing NVG.

Our study is limited by its small sample size, and insufficient data about previous eye status. Nevertheless, wide angle fluorescein angiography revealed wide-spread areas of non-perfusion in both the attached and reattached retina. The retinal capillary non-perfusion and resultant retinal ischemia is likely to be the main pathogenic mechanisms of NVI and NVG.

\section{Conclusions}

In eyes with a history of chronic retinal detachment, NVI and NVG can develop due to peripheral retinal capillary obstruction and retinal ischemia, even after achieving reattachment of the retina. We suggest routine follow up examinations for patients who have suffered chronic retinal detachment, especially in eyes with retinal peripheral non-perfusion on fluorescein angiography.

\section{Abbreviations}

IOP

Intraocular pressure

$\mathrm{NVI}$

Neovascularization of the iris

NVA

Neovascularization of the angle

NVG

Neovascular glaucoma

RRD

Rhegmatogenous retinal detachment

VA

Visual acuity

\section{Declarations}

\section{Ethics approval and consent to participate}

This study was approved by the institutional review board (IRB) of Seoul National University Bundang Hospital (IRB no. B-2112-725-105) and followed the tenets of the Declaration of Helsinki. Since all data collected were retrospective, the requirement of the informed consent was waived by the IRB of Seoul National University Bundang Hospital.

Consent for publication 
Not applicable

Availability of data and materials

All data generated or analysed during this study are included in this published article.

\section{Competing interests}

The authors declare that they have no competing interests

Funding

This work was supported by the National Research Foundation of Korea (NRF) grant No. 2020R1F1A1072795 funded by the Korea government (MSIP).

Authors' contributions

SJW designed the study. DIK collected the data, performed the analysis and interpretation. DIK drafted the manuscript. SJW revised the manuscript.

Acknowledgements

Not applicable

\section{References}

1. Sivak-Callcott JA, O'Day DM, Gass JD, Tsai JC: Evidence-based recommendations for the diagnosis and treatment of neovascular glaucoma Ophthalmology 2001, 108(10):1767-1776, quiz1777, 1800.

2. Tolentino Fl, Lapus JV, Novalis G, Trempe CL, Gutow GS, Ahmad A: Fluorescein angiography of degenerative lesions of the peripheral fundus and rhegmatogenous retinal detachment. Int Ophthalmol Clin 1976, 16(1):13-29.

3. Hayreh SS: Neovascular glaucoma. Prog Retin Eye Res 2007, 26(5):470-485.

4. Cardillo Piccolino F: Vascular changes in rhegmatogenous retinal detachment. Ophthalmologica 1983, 186(1):17-24.

5. Cunha-Vaz JG, Fonseca JR, Vieira R: Retinal blood flow in retinal detachment. Mod Probl Ophthalmol 1979, 20:89-91.

6. Dobbie JG: Circulatory changes in the eye associated with retinal detachment and its repair. Trans Am Ophthalmol Soc 1980, 78:503-566.

7. Rasier R, Gormus U, Artunay O, Yuzbasioglu E, Oncel M, Bahcecioglu H: Vitreous levels of VEGF, IL-8, and TNF-alpha in retinal detachment. Curr Eye Res 2010, 35(6):505-509.

8. Bonnet M: Peripheral neovascularization complicating rhegmatogenous retinal detachments of long duration. Graefes Arch Clin Exp Ophthalmol 1987, 225(1):59-62.

9. Tanaka S, Ideta H, Yonemoto J, Sasaki K, Hirose A, Oka C: Neovascularization of the iris in rhegmatogenous retinal detachment. Am J Ophthalmol 1991, 112(6):632-634.

10. Ghazi NG, Green WR: Pathology and pathogenesis of retinal detachment. Eye (Lond) 2002, 16(4):411-421.

\section{Figures}




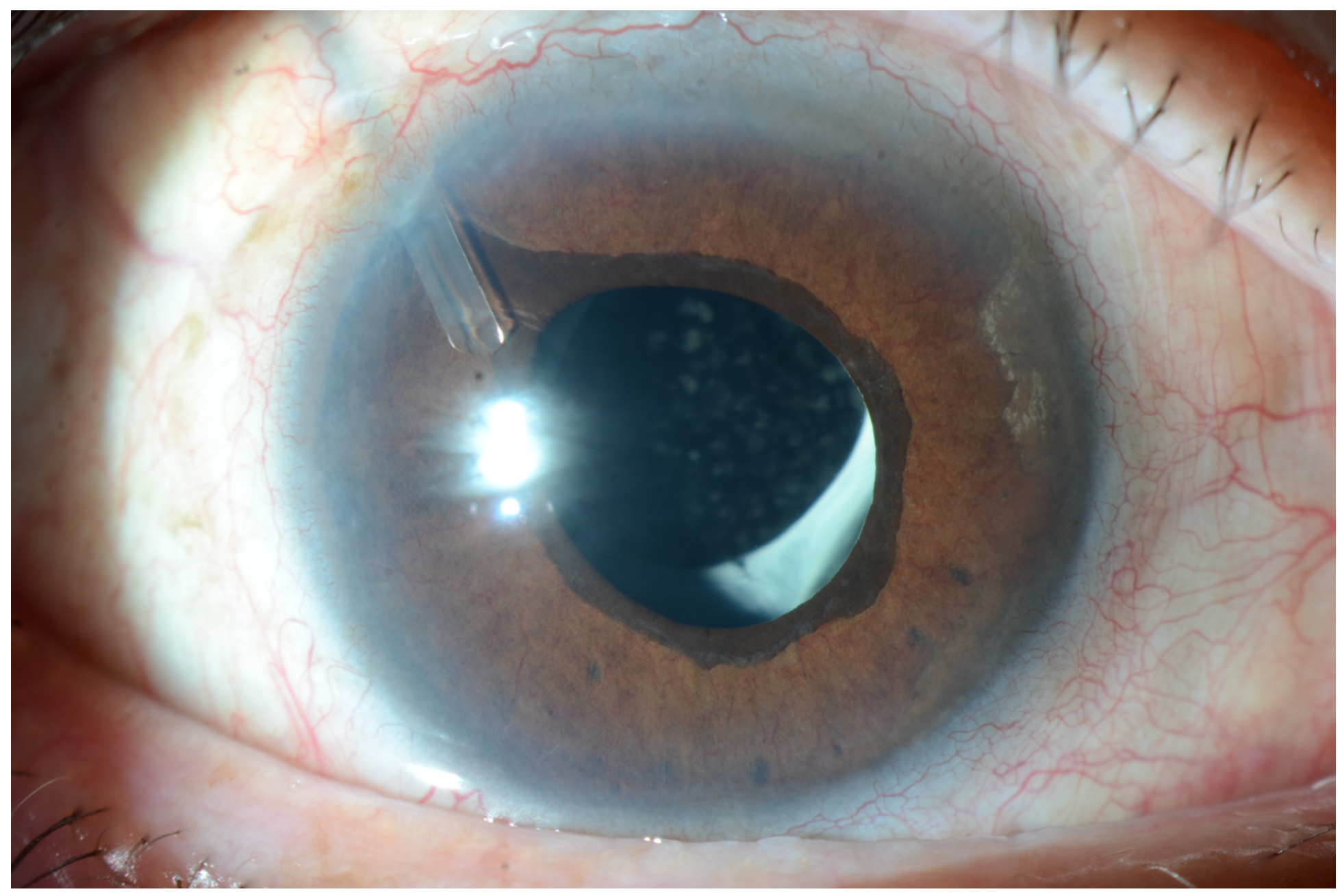

\section{Figure 1}

Anterior segment photography of Case 1, Neovascularization of the iris did not regress even after achieving reattachment of the detached retina. Ahmed valve implantation was performed to control the high intraocular pressure. 


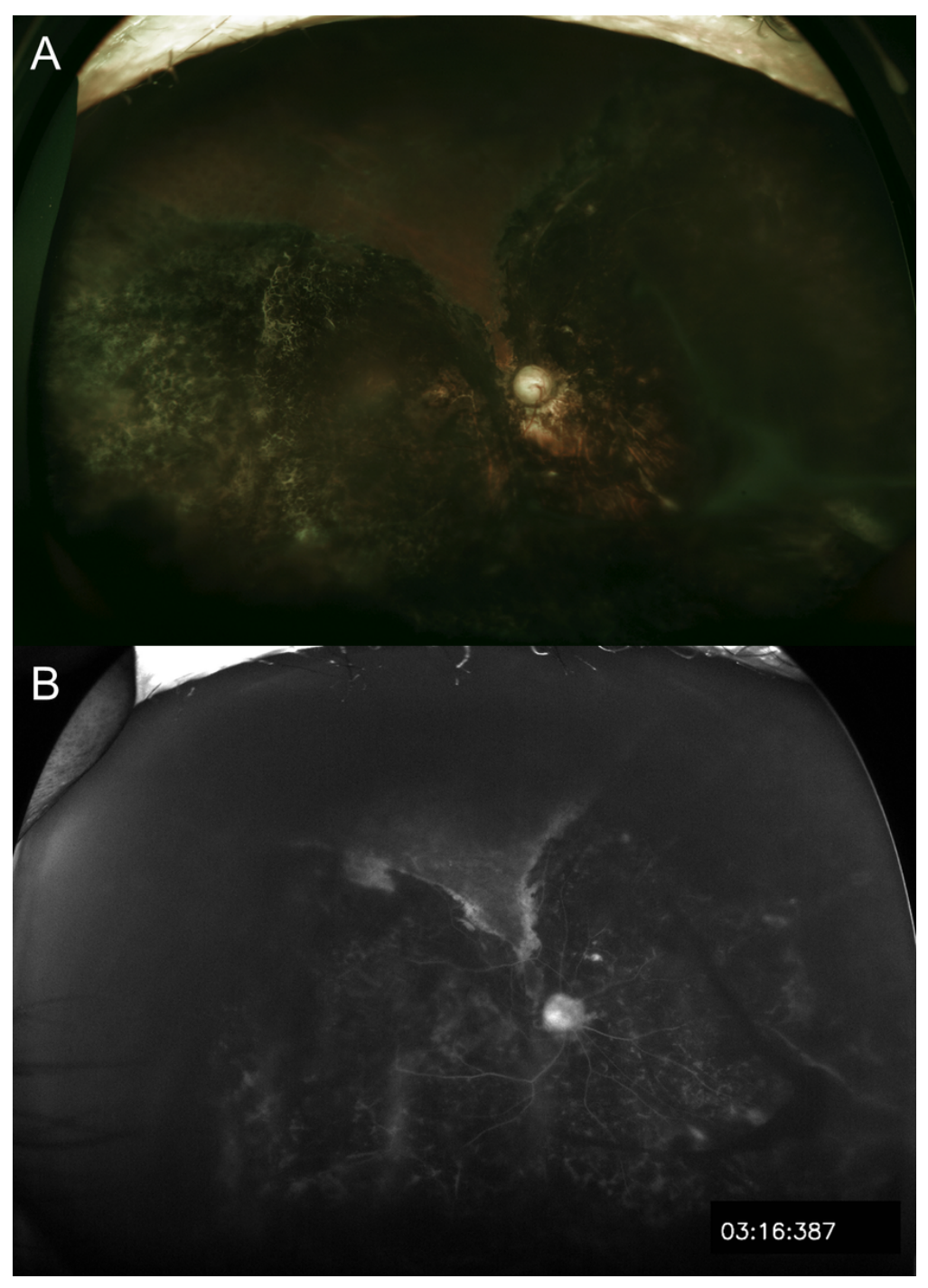

\section{Figure 2}

Case 1. A. Wide-angle fundus photography. The spontaneously reattached retina, which appears as chorioretinal degeneration, covered most of the fundus, whereas the normal-looking, non-detached retina covered only a small superior area. B. Wide-angle fluorescein angiography. Wide areas of nonperfusion were observed in the reattached retina as well as in the portion of the retina that had not been previously detached. 


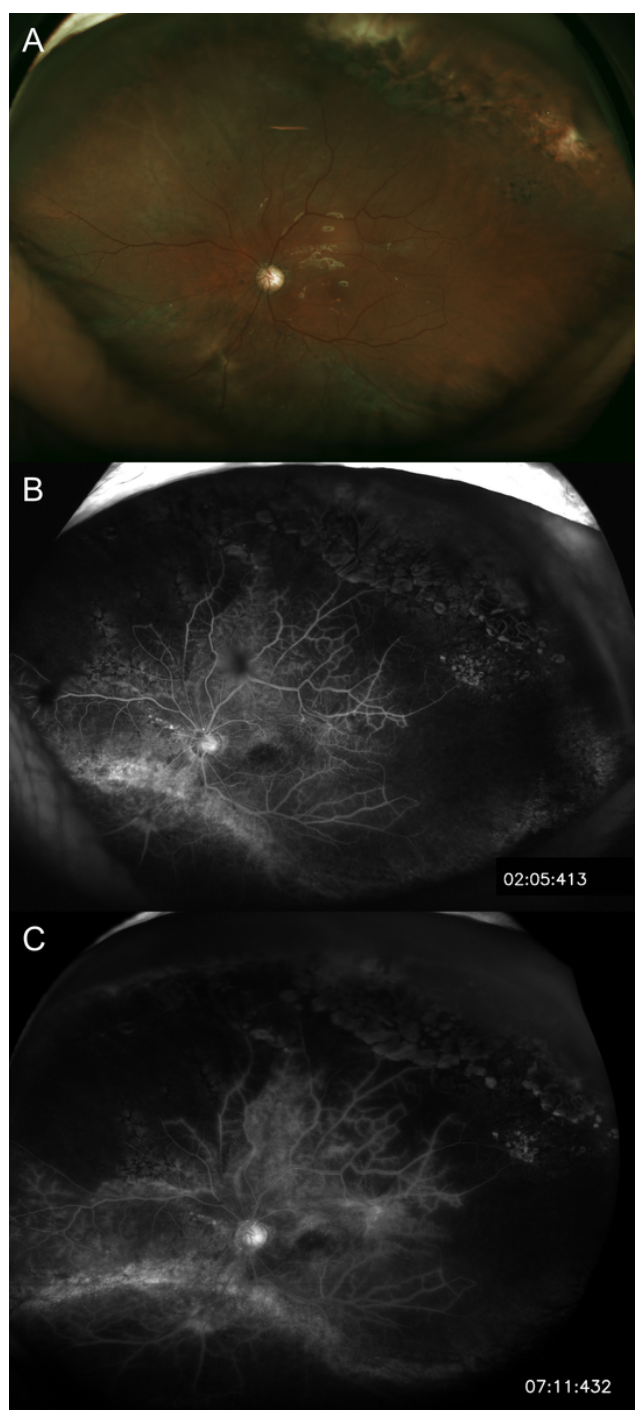

\section{Figure 3}

Case 8. A. Wide-angle fundus photography. This eye had total retinal detachment. The retina was reattached after scleral buckling in the superior area. There were laser scars from panretinal photocoagulation to prevent the development of neovascular glaucoma. B. Wide-angle fluorescein angiography. Wide avascular and non-perfusion areas were observed in the reattached retina. C. Late phase wide-angle fluorescein angiography. Diffuse leakage from retinal vessels indicates severe ischemia. 